\title{
Cultura intraemprendedora e innovación: un estudio empírico en las MIPYME turísticas colombianas *
}

Intra-entrepreneurial culture and innovation: an empirical study in the colombian tourism micro and SMES

Culture intra-entrepreneuriale et innovation: une étude empyrique des petites et moyennes entreprises touristiques colombiennes

Edgar J. Gálvez Albarracín edgar.galvez@correounivalle.edu.co Universidad del Valle, Sede San Fernando. Calle 4B No.36-0o Edificio 124 oficina 3016, Cali-Colombia.

Doctor en Economía y Gestión de Empresas, Universidad Politécnica de Cartagena-España. Magister en Creación de Empresas y Gestión de Proyectos Innovadores, Universidad Montesquieu Bordeaux IV-Francia. Administrador de Empresas, Universidad del Valle, Colombia. Profesor de la Facultad de Ciencias de la Administración, Universidad del Valle, Colombia.

Artículo de investigación científica y tecnológica Según Clasificación Colciencias

Fecha de recepción: agosto 30 de 2011

Fecha de corrección: diciembre 052011

Fecha de aprobación: diciembre 20 de 2011

\section{Resumen}

El objeto de este trabajo es verificar empíricamente la relación existente entre la cultura de intraemprendimientoy la innovación en las empresas. Para ello se realizó un estudio empírico con 68 MIPYME $^{\star \star}$ del sector turístico de Colombia. Los resultados muestran que las prácticas asociadas al intraemprendimiento ejercen influencia positiva sobre la innovación de las MIPYME, y en especial, sobre la de sus procesos productivos y su gestión. Los hallazgos tienen implicaciones para los empresarios ya que les permite comprobar la utilidad del emprendimiento corporativo para el desarrollo de sus organizaciones. Muestra además al sector público, la academia y a las entidades de fomento, la importancia de impulsar programas de investigación, formación y asesoría en el campo del intraemprendimiento, para lograr mayor innovación y competitividad empresarial, lo cual puede redundar a su vez en mayor desarrollo regional y nacional.

Palabras clave: cultura intraemprendedora, innovación, MIPYME, turismo, desarrollo, competitividad, investigación empírica cuantitativa.

Clasificación JEL: M14

* Este artículo de investigación presenta parte de los resultados de la tesis Cultura, innovación, intraemprendimiento y rendimiento en las MIPYME de Colombia , que el autor desarrolló en el marco del doctorado en Economía y Gestión de Empresas de la Universidad Politécnica de Cartagena, España.

** El término MIPYME es generalmente utilizado para agrupar a las micros, pequeñas y medianas empresas. 
Intra-entrepreneurial culture and innovation: an empirical study in the colombian tourism micro and SMES

Cultura intraemprendedora e innovación: un estudio empírico en las MIPYME turísticas colombianas

\section{Culture intra-entrepreneuriale et} innovation: une étude empyrique des petites et moyennes entreprises touristiques colombiennes

\section{Abstract}

This work seeks to empirically verify the relationship between the intra-entrepreneurial culture and innovation in companies. An empirical study was conducted with $68 \mathrm{MI}$ CRO and SMEs from the Colombian tourism sector. The results show that practices associated to intra-entrepreneurship exert positive influence on innovation in MICRO and SMEs and, especially, on innovation in their production processes and management. The findings have implications for entrepreneurs, given that they permit them to test the usefulness of corporate entrepreneurship for the development of their organizations. These findings also show to the public sector, academia, and development entities the importance of promoting research, formation, and advisory programs in the field of intra-entrepreneurship to achieve greater innovation and business competitiveness, which may at the same time result in greater regional and national development.

Keywords: intra-entrepreneurial culture, innovation, MICRO and SMEs, tourism, development, competitiveness, quantitative empirical research.

\section{Culture intra-entrepreneuriale et innovation: une étude empyrique des petites et moyennes entreprises touristiques colombiennes}

\author{
Cultura intraemprendedora e innovación: \\ un estudio empírico en las MIPYME \\ turísticas colombianas \\ Intra-entrepreneurial culture and \\ innovation: an empirical study in the \\ colombian tourism micro and SMES
}

\section{Résumée}

Ĺobjectif de ce travail est de vérifier empiriquement la relation existante entre la culture intra-entrepreneuriale et l'innovation des entreprises. Pour cella on a réalisé une étude empirique avec 68 PME du secteur du tourisme en Colombie. Les résultats montrent que les pratiques associées à l'entrepreneuriat exercent une influence positive sur l'innovation des PME, et spécialement, sur I'innovation des processus productifs et de gestion. Les résultats ont des implications positives pour les chefs d'entreprise parce qu'ils leur permettent de vérifier l'utilité de l'entrepreneuriat corporatif pour le développement des organisations. Ils présentent aussi le secteur public, l'académie et les entités de promotion. Ils renforcent l'importance de stimuler des programmes de recherche, de formation et de conseil dans le domaine de l'intra-entrepreneuriat, pour atteindre un degré plus profond d'innovation et de compétitivité entre entreprises, ce qui peut contribuer au développement régional et national.

Mots clef: culture intra-entrepreneuriale, innovation, PME, tourisme, développement, compétitivité, recherche empirique quantitative. 


\section{Cultura intraemprendedora e innovación: un estudio empírico en las MIPYME turisticas colombianas}

\section{Introducción}

El emprendimiento corporativo o intraemprendimiento representa una actividad emprendedora llevada a cabo dentro de las organizaciones (Trujillo y Guzmán, 2008; Antoncic y Hsrich, 2003), lo que puede traducirse en el desarrollo de proyectos empresariales innovadores y nuevos negocios para la empresa (Varela e Irizar, 2009).

La innovación, como proceso que utiliza la tecnología existente para crear o mejorar un producto, un proceso o un sistema, busca generar, asimilar o explotar con éxito una novedad en los ámbitos económico y social (Medina y Espinosa, 1994). Es así como el Manual de Oslo (OECD y EUROSTAT, 2005) señala que innovar es introducir un nuevo o significativamente mejorado producto (bien o servicio) al mercado, o un nuevo proceso, método de comercialización o de organización, en las prácticas internas de la empresa o en sus relaciones externas.

La cultura emprendedora o de intraemprendimiento es, por lo tanto, aquel tipo de cultura organizacional que a diferencia de la "tradicional", ofrece a los empleados la posibilidad de encontrar oportunidades de innovar, $y$, a la vez, de satisfacer los deseos de sentirse propietarios de sus proyectos internos, sin tener que abandonar la empresa (Garzón, 2004).

A nivel internacional se han realizado trabajos empíricos que relacionan el intraemprendimiento con la innovación organizacional, pudiéndose destacar los de Kuz (2010), Lee et al. (2008), Khazanchi et al. (2007), Manimala (2006), Amo y Kolvereid (2005), Samuelsson y Dahlqvist (2005), Zhao (2005), Garzón (2004), Martins y Terblanche (2003) y el de Claver et al. (1998). Sin embargo estos estudios se han centrado en las grandes corporaciones y no han abordado las micros, pequeñas y medianas empresas, no obstante a que "generan un alto porcentaje del empleo y realizan una considerable parte de la producción y el comercio a nivel mundial" (Julien, 2003).
Esta investigación contribuye a llenar el vacio de literatura señalado, respondiendo a la siguiente pregunta: ¿Las MIPYME con mayor cultura intraemprendedora son más innovadoras? Para ello se realiza un estudio empírico mediante la entrevista a 68 gerentes de hoteles, agencias de viaje y restaurantes MIPYME de Colombia.

Se ha escogido el sector turístico porque en los últimos años a nivel mundial es uno de los de más rápido crecimiento en generación de divisas y empleo (World Economic Forum, 2009), constituyéndose para Colombia en su tercer renglón de exportaciones después del petróleo y el carbón, por lo que el gobierno nacional ha decidido apoyarlo a través de sus políticas públicas (CONPES ${ }^{1}$, 2005).

El documento está estructurado de la siguiente forma: en primer lugar se revisan los estudios empíricos previos y se plantea la hipótesis de investigación, en segundo lugar se expone la metodología usada lo cual incluye la obtención de la muestra, la recolección de datos y la medición de variables. En tercer lugar se presentan los resultados y su discusión, y finalmente se exponen las conclusiones, las limitaciones del trabajo y se señalan posibles investigaciones futuras.

\section{Estudios empíricos previos}

Como se puede apreciar a continuación, la relación entre intraemprendimiento e innovación organizacional ha sido objeto de diversos trabajos empíricos, que la han estudiado de manera exclusiva o relacionandola con otras variables, en diferentes contextos geográficos y culturales:

En los Estados Unidos de América, Kuz (2010) estudia el intraemprendimiento y la innovación en empresas de alta tecnología, vinculando estos factores al papel desempeñado por sus líderes de mayor rango. Su estudio muestra que para que la empresa sea más innovadora el líder debe fomentar prácticas como la generación de ambientes de tra- 
bajo colaborativos, apoyar la innovación abierta a través de concursos de ideas, vincular el intraemprendedor a los procesos innovadores, y conducir la empresa hacia negocios de más actualidad.

Lee et al. (2008) en un estudio con empresas de Taiwan relacionan el aprendizaje y los comportamientos organizacionales asociados al intraemprendimiento y la innovación. Hallan una relación positiva y significativa entre la cultura organizacional intraemprendedora y la innovación, demostrando que las empresas con este tipo de cultura son más innovadoras en productos y a nivel gerencial.

Explorando como los valores organizacionales impactan en el proceso de innovación y, en especial, la manufactura tecnológicamente avanzada AMT, Khazanchi et al. (2007) encontraron que los valores relacionados con la flexibilidad (considerada por muchos autores como una de las principales carácteristicas de la cultura intraemprendedora), afectan positiva y directamente la innovación y que en la medida que los gerentes y operarios comparten dicha percepción de flexibilidad, el rendimiento de la planta mejora después de la implementación de un sitema de AMT.

Manimala (2006) presentó un estudio realizado en empresas del sector público de la India, cuyo principal objetivo fue identificar las dificultades de la organización para la innovación intraemprendedora. Para cumplir con su proposito revisó 162 proyectos, de los que seleccionó 31 casos altamente innovadores. Entre sus resultados identificó las siguientes dificultades para la innovación intraempresarial: insuficiencia de premios y reconocimiento $(65 \%)$, pobre documentación y mantenimiento de registros ( $58 \%$ ), y falta de formalidad en la formación del equipo ( $81 \%)$.

Amo y Kolvereid (2005) aplicaron un modelo orientado a medir el comportamiento intraemprendedor e innovador en graduados de la Escuela de Negocios de la Universidad de Norway. El estudio que incluyó una muestra 634 individuos ocupantes desde gerencias de niveles alto y medio, hasta cargos de menor rango en diferentes organizaciones, arrojó resultados que sustentan la relación entre la orientación hacia el intraemprendimiento y la renovación definida y alcanzada por la organización.

En un estudio empírico realizado en Suecia, Samuelsson y Dahlqvist (2005) analizaron si las innovaciones intraemprendedoras se presentan más en las empresas nuevas o en las ya establecidas; encuentraron que en promedio la mayoría de emprendimientos innovadores se desarrollan dentro de organizaciones más antiguas, y que los recursos y capacidades que han logrado acumular estas empresas se constituyen en un factor diferenciador al respecto.

Zhao (2005) buscando aportar al entendimiento de la relación entre emprendimiento y la innovación en las organizaciones, realizó un estudio de casos múltiples con seis empresas innovadoras de Australia. Encontró que existe una relación positiva, directa y complementaria entre los factores observados, y que en entornos cambiantes, la combinación de ambos es vital para el éxito organizativo, así mismo, concluyó que el estilo de dirección y, en general, la cultura organizacional son factores que influyen en el comportamiento innovador de la firma.

En Colombia Garzón (2004) realizó un trabajo con las PYMES de Bogotá para observar el papel de los altos intraemprendedores y la cultura intraemprendedora en los procesos de innovación; encontró que los factores que facilitan el trabajo intraemprendedor en las PYMES son en orden jerárquico: el apoyo a las ideas innovadoras de los colaboradores, la identidad y el sentido de pertenencia con el equipo y la empresa, la autonomía, una estructura organizacional flexible, una adecuada relación desempeño-premio para el colaborador y la tolerancia institucional al conflicto.

Martins y Terblanche (2003) estudiaron los elementos de la cultura organizacional que influyen sobre el desarrollo de la creatividad y la innovación en las empresas, obteniendo como resultado que son especialmente el fomento de la generación de ideas y la toma de riesgos, el aprendizaje continuo, el soporte para afrontar el cambio y la competencia, y el apoyo en caso de cometer errores o en casos de conflictos intraorganizacionales.

Claver et al. (1998) en un trabajo que analiza el origen de la innovación tecnológica y las condiciones más importantes para que se produzca en la organización, encuentran que se requiere de: preparación técnica, un factor humano preparado, recursos financieros, pero sobre todo de una cultura organizacional favorable al cambio; y que todos estos factores son 
determinantes para el éxito de la estrategia innovadora de la firma.

Partiendo de los aportes mencionados se plantea la siguiente hipótesis:

Las MIPYME con mayor cultura intraemprendedora son más innovadoras.

\section{Metodología de la investigación}

El presente trabajo de investigación gira en torno a la cultura intraemprendedora y su impacto en la innovación de las MIPYME colombianas, contrastando la hipótesis de investigación previamente presentada.

\subsection{Obtención de la muestra y recolección de datos}

Las empresas objeto de este estudio fueron las MIPYME del sector turístico de Colombia, en particular hoteles, agencias de viajes y restaurantes. Para conformar la muestra se utlizaron las bases de datos de afiliados a sus respectivas agremiaciones: COTELCO ${ }^{2}$ para los hoteles, ANATO $^{3}$ para las agencias de viaje y ACODRES 4 para los restaurantes, identificándose un total de 2012 empresas.

La tabla 1 ilustra la distribución de la muestra por sector de actividad y tamaño de la empresa.

\begin{tabular}{|c|c|c|c|c|c|}
\hline \multicolumn{2}{|c|}{$\begin{array}{l}\text { Tamaño de la } \\
\text { empresa / No. de } \\
\text { trabajadores }\end{array}$} & $\begin{array}{c}\text { Micro de } \\
\text { 1 a } 10\end{array}$ & $\begin{array}{c}\text { Pequeña de } \\
11 \text { a } 50\end{array}$ & $\begin{array}{l}\text { Mediana } \\
51 \text { a } 200\end{array}$ & $\begin{array}{c}\text { Número } \\
\text { de empresas }\end{array}$ \\
\hline \multirow{4}{*}{ 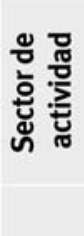 } & Hoteles & 11 & 15 & 3 & 29 \\
\hline & $\begin{array}{c}\text { Agencias de } \\
\text { viajes }\end{array}$ & 10 & 12 & 2 & 24 \\
\hline & Restaurantes & 8 & 6 & 1 & 15 \\
\hline & Total & 29 & 33 & 6 & 68 \\
\hline
\end{tabular}

Fuente: elaboración propia.

Las empresas se seleccionaron de manera aleatoria, se pidieron 300 citas y 68 firmas aceptaron participar en la investigación. Esto representa un margen de error de 9.5 puntos con un margen de confianza del $95 \%$. Los datos se recolectaron mediante una encuesta especialmente estructurada para esta investigación a partir de la literatura disponible; el instrumento fue aplicado directamente por los miembros del equipo de investigación. El trabajo de campo se realizó entre los meses de noviembre del 2010 y enero del 2011.

\subsection{Medición de variables}

\subsubsection{Cultura intraemprendedora}

Para medir esta variable, a partir de una amplia revisión de trabajos teóricos y empíricos, se desarrolló un cuestionario que analiza cinco elementos: 1) autonomía para los colaboradores, 2) tolerancia al riesgo y a los errores, 3) compensación e incentivos, 4) trabajo en equipo, y 5) soporte de la gerencia y flexibilidad en la estructura corporativa. La tabla 2 presenta el cuestionario aplicado, mostrando cómo cada pregunta se soporta en los aportes de los autores revisados.

Las preguntas se presentan en una escala Likert de 5 puntos, donde 1 significa que el encuestado está nada de acuerdo y 5 que está totalmente de acuerdo. De igual manera en la tabla señalada se muestra cómo el estadístico Alpha de Cronbach valida la escala utilizada. El Alpha de Cronbach es habitualmente utilizado como medida de fiabilidad respecto a un conjunto de dos o más ítems de un constructo (en donde los valores van desde o a 1 , considerandose de 0.60 hasta 0.70 el límite inferior de aceptabilidad (Hair et al. 1999).

\footnotetext{
2. COTELCO es la Asociación Hotelera de Colombia. www.cotelco.org

3. ANATO es la Asociación Colombiana de Agencias de Viaje y Turismo. www.anato.org

4. ACODRES es la Asociación Colombiana de la Industria Gastronómica. www.acodres.com.co
} 


\section{Tabla 2. Elementos de la cultura intraemprendedora}

\section{Variable considerada}

Autonomía para los colaboradores

¿En su organización es permitido que los funcionarios inicien algunos proyectos sin tener que consultarlo con los directivos?

¿En su organización se persuade más que se obliga a los funcionarios para que participen en nuevos proyectos?

¿Los colaboradores pueden tomar decisiones autónomamente en el desarrollo de los proyectos institucionales?

Tolerancia al riesgo y a los errores

¿En su empresa las personas que toman riesgos son valoradas y bien vistas, aunque algunas veces se equivoquen? ¿La empresa estimula que los colaboradores tomen riesgos calculados?

Compensación e incentivos

Trabajo en equipo

$$
\begin{aligned}
& \text { ¿En la organización está bien vista la generación } \\
& \text { espontanea de equipos para asuntos laborales? } \\
& \text { ¿La empresa con frecuencia fomenta el trabajo entre } \\
& \text { colaboradores de diferentes áreas y/o niveles } \\
& \text { jerárquicos? }
\end{aligned}
$$

\section{¿En la empresa a las personas que generan y/o impulsan proyectos novedosos se les reconoce públicamente? \\ ¿La empresa recompensa económicamente a los colaboradores que generan nuevas ideas o proyectos?}

Soporte de la gerencia y flexibilidad en la estructura corporativa

¿Es frecuente que las directivas se la jueguen por los proyectos de sus colaboradores?

¿Existe un programa institucional para fomentar la iniciativa de los colaboradores?

¿Es frecuente que en la empresa se apoyen nuevos proyectos así no hayan estado en el plan estratégico o el presupuesto previamente aprobado? ¿Las directivas permiten que los colaboradores usen parte de su tiempo laboral para la planeación y/o el desarrollo de los proyectos auto iniciados?
Autores y enfoques de referencia

Varela e Irizar, 2009; Moriano et al., 2009; Garzón, 2005; Sathe, 2003; Hornsby, Kuratko y Zahra, 2002; Ross, 1987; Girard, 1989, Pinchot III,1985.

Varela e Irizar, 2009; Garzón, 2005; Chen et al., 2005; Kyriakopoulos et al., 2004; Antoncic, 2003; Morris y Kuratko, 2002; Wood, 2004; Hornsby et al., 2002; Girard, 1989; Ross, 1987; Pinchot III, 1985.

Varela e Irizar, 2009; Moriano et al., 2009; Van Brusel y Ulijn 2008; Manimala, 2006; Chrístensen, 2005; Garzón, 2005; Wood, 2004; Hornsby et al., 2002; Pinchot III, 1985.

Varela e Irizar, 2009; Manimala, 2006; Garzón, 2005; Kyriakopoulos et al., 2004; Sathe, 2003; Morris et al., 1993; Ross, 1987; Pinchot III, 1985 .

Varela e Irizar, 2009; Moriano et al., 2009; Chen et al., 2005; Garzón, 2005; Wood, 2004; Kyriakopoulos et al., 2004; Martins y Terblanche, 2003; Sathe, 2003; Hornsby et al., 2002; Pinchot III, 1985

\subsubsection{Variable de innovación}

Para medir el grado de innovación de las empresas existen dos enfoques (Hughes, 2001), uno objetivo que utiliza datos de tipo cuantitativo como el número de patentes, la innovación en productos (número de nuevos productos) o en los procesos (costes de inversión); y un enfoque subjetivo, basado en la percepción del gerente o propietario de la empresa sobre su actividad innovadora. En el caso de las MIPYME resulta más apropiado el enfoque subjetivo, dado que el enfoque objetivo tiende a subestimar su actividad innovadora (Hughes, 2001). Este enfoque, por ejemplo, es el que se utiliza en el Estudio de la Innovación Armonizada de la Unión Europea.

Para abordar este factor en la investigación, se tuvieron en cuenta los diferentes 
conceptos recopilados en la revisión de trabajos realizada, y en especial el de AECA (1995) que señala que la innovación puede clasificarse en tecnológica y organizacional. Las innovaciones tecnológicas incluyen las novedades significativas en productos y en procesos; la innovación organizacional por su parte corresponde a los cambios introducidos a la estructura administrativa de la empresa, a la comercialización, a la financiación, etc. Esta misma clasificación ha sido usada en diferente trabajos como los de Maldonado et al., 2009; Van Auken et al., 2008; Naranjo et al., 2008.

Para medir el grado de innovación, en productos, procesos y gestión, se utilizó una medida multicriterio de varios ítems por cada tipo de innovación, con una escala Likert de cinco puntos (de $1=$ grado de innovación poco importante a $5=$ grado de innovación muy importante). La variable innovación en productos está compuesta por la media aritmética de dos ítems: 1 ) cambios o mejoras en productos o servicios existentes, y 2) comercialización de nuevos productos. La variable innovación en procesos está compuesta por la media aritmética de dos ítems: 1) mejoras o cambios en los procesos productivos, y 2) adquisición de nuevos equipos. Y la variable innovación en gestión está compuesta por la media aritmética de tres ítems: 1) gestión de dirección, 2) compras y aprovisionamientos, y 3) comercialización y ventas.

Para validar estas medidas se verifica la fiabilidad de las escalas a través del estadístico Alpha de Cronbach (tabla 3). El valor del estadístico indica la validez de la escala utilizada.
Las estimaciones se realizan a partir de regresiones lineales múltiples por MCO. Inicialmente se comprobó en todos los modelos que los regresores presentan un factor de inflación de la varianza (VIF) menor a 1.831, por lo que se descartó la presencia de multicolinealidad.

\subsection{Modelo analizado}

Para contrastar la hipótesis planteada en este trabajo se consideró el siguiente modelo de investigación:

Las MIPYME con mayor cultura intraemprendedora son más innovadoras.

$$
\begin{aligned}
\text { Innovación } n_{i}= & b_{\circ}+b_{1} \text { Auton }_{i}+b_{2} \text { Toler }_{i}+b_{3} \text { Comp }_{i} \\
& +b_{4} \text { Trab }_{i}+b_{5} \text { Sop }_{i}+\varepsilon_{i}
\end{aligned}
$$

\section{Dependiente Innovación}

Innovación en productos

Innovación en procesos

Innovación en gestión

Innovación global

\section{Explicativas (Factores de Intraemprendi- miento) \\ Toler: Tolerancia al riesgo y a los errores \\ Comp: Compensación e incentivos \\ Trab: Trabajo en equipo \\ Sop: Soporte de la gerencia y flexibilidad en la estructura corporativa}

\section{Resultados y discusión}

La tabla 4 presenta los datos descriptivos de las variables analizadas. Puede observarse que en promedio las MIPYME del sector turístico colombiano son más innovadoras que intraemprendedoras. En cuanto a las prácticas relacio-

\section{Tabla 3 . Tipos de innovación}

\section{Indique cuál ha sido el grado de innovación realizado en su empresa en los dos últimos años: (1= Poco importante, $5=$ Muy importante)}

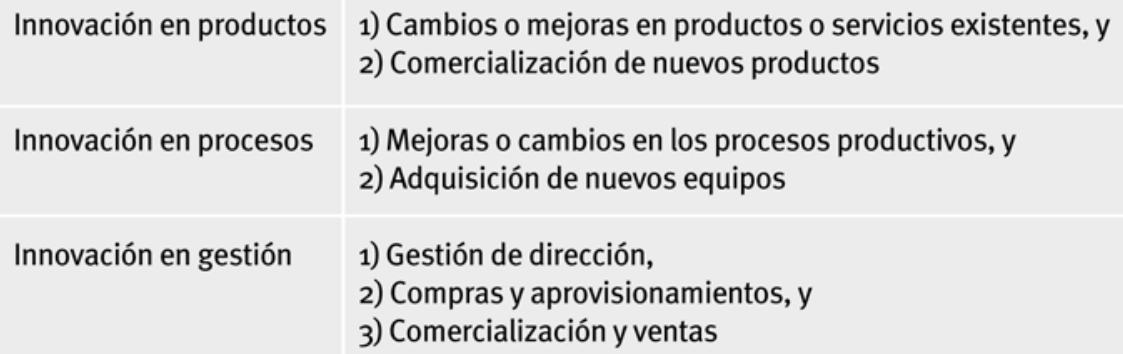


nadas con la cultura de intraemprendimiento se puede apreciar que los factores que presentan mayores valores son los de trabajo en equipo, compensación e incentivos y tolerancia al riesgo y a los errores, todos ellos superiores a 3, mientras que el soporte de la gerencia y la flexibilidad en la estructura corporativa, así cómo la autonomía obtienen valores inferiores. De igual manera se muestra que estas empresas desarrollan más innovaciones de productos/servicios y menos en gestión, aunque en todos los casos los valores se sitúan por encima de 3 (Tabla 4)

\begin{tabular}{|l|l|l|l|l|}
\hline \multicolumn{1}{|c|}{ Tabla 4. Descriptivos de las variables } & Media & $\begin{array}{c}\text { Desviación } \\
\text { Estándar }\end{array}$ & Min & Max \\
\hline Cultura Intraemprendedora & & & & \\
\hline - Autonomía & 2.78 & 0.89 & 1 & 4 \\
\hline - Tolerancia al riesgo y a los errores & 3.04 & 1.04 & 1 & 5 \\
\hline - Compensación e incentivos & 3.26 & 1.24 & 1 & 5 \\
\hline - Trabajo en equipo & 3.28 & 1.03 & 1 & 5 \\
\hline - Soporte de la gerencia y flexibilidad en la & 2.56 & 1.06 & 1 & 4 \\
\hline estructura corporativa & & & & \\
\hline Innovación & & & & \\
\hline - Productos/servicios & 3.69 & 1.31 & 0 & 5 \\
\hline - Procesos & 3.42 & 1.39 & 0 \\
\hline - Gestión & 3.10 & 1.44 & 5 \\
\hline
\end{tabular}

Por su parte, la tabla 5 muestra la relación entre los cinco factores de cultura de intraemprendimiento: autonomía, tolerancia al riesgo y a los errores, compensación e incentivos, trabajo en equipo, soporte y flexibilidad en la estructura corporativa, más el agregado de dichos factores que constituye el intraemprendimiento global; frente a los tipos de innovación considerados: productos, procesos y gestión, más su agregado que representa la innovación global. Los resultados se describen a continuación:

Efectos de la autonomía, la tolerancia al riesgo y a los errores, y la compesación e incentivos sobre la innovación

No se encontró un impacto significativo de estos tres factores de la cultura intraemprededora sobre los diferentes tipos de innovación. Estos resultados difieren de los del estudio de Manimala (2006), en el cual la compensación aparece como un factor importante para que se produzcan innovaciones en la empresa, y con los de Garzón (2004) quien encuentra que la compensación y la autonomía tienen el mismo efecto. Los resultados en este aspecto y sus diferencias con los otros señalados, pueden deberse a que como se pudo apreciar en los análisis descriptivos, las MIPYME turísticas de Colombia, hacen una relativamente baja aplicación de las practicas asociadas a estos factores de intraemprendimiento, por lo cual no alcanzan a impactar su desempeño innovador.

\section{Efectos del trabajo en equipo sobre la innovación}

El trabajo en equipo como factor de la cultura de intraemprendimiento tiene asociados coeficientes positivos y significativos para sus relaciones con la innovación en gestión y la innovación global $\left(0,347^{\star \star \star}, 0,286^{\star \star}\right.$ respectivamente). Ello indica que en la medida en que se fomenta el trabajo entre personas de diferentes áreas y niveles jerárquicos se genera en la organización una mayor innovación en los sistemas de dirección y gestión, compras y aprovisionamiento, comercialización y ventas; de igual manera que en términos generales, la empresa se vuelve más innovadora. Estos resultados confirman los de Kuz (2010) y los de Garzón (2004), quienes encuentran que un ambiente colaborativo y la identificación del empleado con la empresa y el equipo de trabajo, son fundamentales para que se dé la innnovación corporativa.

Efectos del soporte de la gerencia y la flexibildiad de la estructura corporativa sobre la innovación

Este factor de la cultura intraemprendedora tiene asociado un coeficiente positivo y significativo en su relación con la innovación en procesos $\left(0,315^{\star \star \star}\right)$. Esto significa que cuando en las MIPYME se apoyan las iniciativas de los colaboradores, se asignan recursos a los proyectos presentados por estos y se les permite utilizar parte de su tiempo laboral en ellos, mejora la innovación en los procesos de producción, y el aprovechamiento de los equipos adquiridos. Estos hallazgos confirman los de Kuz (2010), 
los de Garzón (2004) y los de Martins y Terblanche (2003), en cuanto a que este factor de intraemprendimiento fomenta la innovación empresarial; pero en especial, ratifican los de Khazanchi et al. (2007) quienes encuentran que la flexibilidad fomenta la innovación en procesos productivos. Por otra parte los resultados de este trabajo difieren de los de Lee et al. (2008) quienes sí encuentran un efecto positivo del soporte de la gerencia y la flexibilidad de la estructura corporativa sobre la innovación en gestión y en productos/servicios.

Se comprueba la válidez global de los modelos que relacionan el intraemprendimiento con la innovación en procesos, gestión e innovación global, puesto que sus $\mathrm{F}$ son significativas $\left(7,262^{\star \star}\right.$, $9,029^{\star \star \star}, 5,896^{\star \star}$ respectivamente).

Efectos del intraemprendimiento global sobre la innovación

Para el agregado de los diferentes factores de cultura intraemprendedora o intraemprendimiento global, se encuentran coeficientes positivos y significativos en su relación con la innovación en pro- cesos, en gestión y en innovación global $\left(0,249^{\star \star}\right.$, $0,275^{\star \star}, 0,254^{\star \star}$ respectivamente). Esto indica que si la organización es más intraemprendedora, mejora su desempeño innovador en cuanto a procesos de: producción o servicios, aprovechamiento de los equipos adquiridos, dirección, gestión, compras y aprovisionamiento, comercialización y ventas; así como a nivel general. Estos resultados confirman los de Amo y Kolvereid (2005) y los de Zhao (2005), quienes hallan que las prácticas asociadas a la cultura intraemprendedora influyen positivamente sobre la renovación corporativa y en el desempeño innovador de la compañía.

Se encuentra validez global de los modelos para las mismas relaciones: procesos, gestión e innovación global ya que sus $\mathrm{F}$ tienen valores sigficativos $\left(4,358^{\star \star}, 5,414^{\star \star}, 4,549^{\star \star}\right.$ respectivamente).

De acuerdo con los resultados presentados, se puede confirmar la hipótesis planteada en este trabajo, pero parcialmente, dado que como se indicó previamente las prácticas asociadas a la cultura de intraemprendimiento no tuvieron efectos significativos sobre la innovación en productos (Tabla 5)

\begin{tabular}{|c|c|c|c|c|}
\hline \multirow{2}{*}{ Intraemprendimiento } & & Innovación & & \multirow{2}{*}{$\begin{array}{l}\text { Innovación } \\
\text { Global }\end{array}$} \\
\hline & Productos & Procesos & Gestión & \\
\hline Autonomía & n.s & n.s & n.s & n.s \\
\hline Tolerancia al riesgo & n.s & n.s & n.s & n.s \\
\hline Compensación & n.s & n.s & n.s & n.s \\
\hline Trabajo en equipo & n.s & n.s & $\begin{array}{c}0,347^{\star \star \star} \\
(3,005)\end{array}$ & $\begin{array}{c}0,286^{\star \star} \\
(2,248)\end{array}$ \\
\hline $\begin{array}{l}\text { Soporte de gerencia y flexibilidad en la } \\
\text { estructura organizacional }\end{array}$ & n.s & $\begin{array}{l}0,315^{\star \star \star} \\
(2,695)\end{array}$ & n.s & n.s \\
\hline $\mathrm{F}-\mathrm{R}^{2}$ ajustado & n.s & $\begin{array}{c}7,262^{\star \star \star} \\
0,085\end{array}$ & $\begin{array}{l}9,029^{\star \star \star} \\
0,107\end{array}$ & $\begin{array}{l}5,896^{\star *} \\
0,068\end{array}$ \\
\hline Intraemprendimiento global & n.s & $\begin{array}{l}0,249^{\star \star} \\
(2,088)\end{array}$ & $\begin{array}{l}0,275^{\star \star} \\
(2,327)\end{array}$ & $\begin{array}{c}0,254^{* *} \\
(2,133)\end{array}$ \\
\hline $\mathrm{F}-\mathrm{R}^{2}$ ajustado & n.s & $\begin{array}{l}4,358^{\star \star} \\
0,248\end{array}$ & $\begin{array}{c}5,414^{\star \star} \\
0,062\end{array}$ & $\begin{array}{l}4,549^{\star \star} \\
0,050\end{array}$ \\
\hline
\end{tabular}

\section{Conclusiones e implicaciones}

En esta investigación se ha analizado el efecto de la cultura intraemprendedora o de intraemprendimiento sobre la innovación de las MIPYME, utilizando una muestra de $68 \mathrm{em}$ presas. De esta forma se contribuye a ampliar el conocimiento del tejido empresarial colombiano, donde este tipo de estudios empíricos es escaso.

Según los hallazgos de este trabajo, las MIPYME del sector turístico en Colombia tienen un promedio apenas aceptable de utilización de las prácticas asociadas a la cultura de intraem- 
prendimiento (2,98 sobre 5); aún así el impacto de estas es significativo en su comportamiento innovador global, pero en especial en sus procesos productivos y en su gestión. Lo señalado demuestra que el emprendimiento corporativo debe ser considerado como una estrategia a implementar por aquellas MIPYME que necesiten o deseen mejorar su nivel general de innovación, y en particular el de su operación productiva, gerencial y comercial.

Qué el trabajo en equipo se presente en esta investigación como el factor de intraemprendimiento con mayor impacto positivo sobre la innovación de las empresas, reafirma la importancia de estimular la sinergia que se produce al combinar la capacidad creativa de los colaboradores de diferentes niveles y/o departamentos.

Por su parte que el intraemprendimiento global haya mostrado impacto positivo en la mayor parte de los tipos de innovación observados, muestra que son justificados los esfuerzos que se hagan para implementar en las MIPYME turísticas las diferentes prácticas asociadas a la cultura de intraemprendimiento, ya que ello mejora su desempeño innovador $y$, por ende, su nivel competitivo; teniendo eso si que buscarse nuevas prácticas de intraemprendimiento que logren estimular su innovación en productos/ servicios.

La contribución de este trabajo al contexto de la MIPYME es relevante, ya que en Colombia los prestadores turísticos son principalmente micro y pequeñas empresas, que requieren alcanzar estándares de calidad y competitividad que les permitan consolidarse como actores de clase mundial (CONPES, 2005).

Los resultados de esta investigación son de importancia ya que proporcionan pautas a los empresarios y gerentes acerca de las prácticas de intraemprendimiento que deberían impulsar para mejorar el desempeño innovador de sus organizaciones. A las entidades públicas y privadas de fomento de la actividad empresarial les ofrecen nuevas perspectivas para reforzar sus programas de intervención en los aspectos de mayor impacto en la innovación de las empresas. La academia puede utilizarlos para orientar sus actividades de investigación, docencia y extensión hacia aquellas que más contribuyan a generar o fortalecer una cultura de intraemprendimiento que estimule la competitividad y el desarrollo de las MIPYME.
La investigación presenta, entre otras, las siguientes limitaciones: el tamaño de la muestra es relativamente pequeño lo que conlleva un margen de error de 9.5 con un margen de confianza del $95 \%$. Aunque este margen es aceptable desde un punto de vista investigativo, hubiera sido deseable no superar un margen de error de 5 puntos. En este sentido, una muestra más amplia hubiera permitido analizar los resultados discriminándolos según el tamaño de las empresas (micro, pequeñas y medianas) y el sector específico de actividad (hoteles, restaurantes, y agencias de viaje). Por otra parte, la encuesta fue aplicada sólamente a los directivos de la empresa, siendo deseable utilizar informantes de diferente nivel para limitar las posibilidades de sesgos perceptuales (Cameron y Quinn, 1999; Naranjo et al., 2008).

Futuros estudios podrían retomar esta línea de investigación para analizar elementos que quedan pendientes, tales como los factores de la cultura intraemprendedora predominantes según el tamaño de las empresas y en cada actividad específica del turismo. De igual manera, se podrían añadir variables de control como la edad de la empresa, la condición de ser o no de tipo familiar, el género del gerente y su grado de escolaridad. También se podrían incluir otros actores del sector turístico, tales como las empresas de transporte terrestre y las de alquiler de vehículos.

\section{Agradecimientos}

El autor agradece el apoyo institucional y económico que para este trabajo ha recibido de la Universidad del Valle en Colombia, Universidad Politécnica de Cartagena en España, y de la Asociación Universitaria Iberoamericana de Postgrado AUIP. De igual manera manifiesta su agradecimiento a los asistentes de investigación Ana María García y Fred Davinson Contreras.

\section{Referencias}

AECA (1995). La innovación en la empresa: factor de supervivencia. Principios de organización y sistemas. Madrid, España: Asociación Española de Contabilidad y Administración de Empresas.

Amo, B. y Kolvereid, L. (2005). Organizational strategy, individual personality and innovation behavior. Journal of Enterprising Culture, 13 (1), pp. 7-19. 
Antoncic, B. (2003). Risk taking in intrapreneurship: Translating the individual level risk aversion into the organizational risk taking. Journal of Enterprising Culture , 11 (1), pp. 1-23.

Antoncic, B. y Hisrich, R. (2003). Clarifying the intrapreneurship concept. Journal of Small Business and Enterprise Development. 10(1), pp. 7-24.

Cameron, K. y Quinn, R. (1999). Diagnosing an changing organizational culture. based on the competing values framework. San Francisco, CA: Prentice Hall Series in Organizational Developement.

Claver, E., Llopis, J., García, D. y Molina, H. (1998). Organizational culture for innovation and new technological behavior. The journal of High Technology Management Research, 9 (1), pp. 55-68.

CONPES. (2005). Documento CONPES 3397 Política Sectorial de Turismo. Bogotá: Consejo Nacional de Política Económica y Social. Departamento Nacional de Planeación. Recuperado el 24 de enero de 2011 de: http://www.dnp.gov.co/ Portals/o/archivos/documentos/Subdireccion/ Conpes/3397.pdf.

Chen, J., Zhu, Z. y Anquan, W. (2005), A System Model for Corporate Entrepreneurship. International Journal of Manpower, 26 (6), pp. 529-545.

Christensen, S. K. (2005). Enabling intrapreneurship: The case of a knowledge-intensive industrial company. European Journal of Innovation Management, 8(3), pp. 305-318.

Garzón, M. A. (2004). La innovación intraemprendedora liderada por los gerentes de las Pymes. Universidad Empresa, 3(6), pp. 74-109.

Garzón, M. A. (2005). Modelo intraemprendedor para la innovación. Bogotá, Colombia: Universidad del Rosario.

Girard, J. (1989). Un leadership transparent et combatiff. Montreal, Canadá: Colloque de la Fondation de L'entrepreneurship.

Hair, J.F., Anderson, R. E., Tatham, R. L. y Black, W. C. (1999). Análisis Multivariante. Madrid, España: Prentice Hall.

Homsby, J. S., Kuratko, D. F. y Zahra, S. A. (2002). Middle managers perception of the internal environment for corporate entrepreneurship: Assessing a measurement scale. Journal of Business Venturing, 17 (3), pp. 253-273.

Hughes, A. (2001). Innovation and Business Performance: Small Entrepreneurial Firms in the UK and the EU. New Economy, n. 8, pp. 157-163.

Julien, P. A. (2003). Las PYME: Balance y perspectiva. Cali, Colombia: Universidad ICESI.

Khazanchi, S., Lewis, M. y Boyer, K. (2007). Innovation-supportive culture: The impact of organizational values on process innovation. Journal of Operations Management, n. 25, pp. 871-884.

Kuz, D. (2010). Exploration of Intrapreneurship and Innovation in Advanced Technology Organizations in the Western United States. Recuperado el 24 De enero de 2011, de: http://gradworks. umi.com/3425617.pdf.

Kyriakopoulos, K., Meulenber, M., y Nilsson, J. (2004). The Impact of Cooperative Structure and Firm Culture on Market Orientation and Performance. Agrobusiness, 20(4), pp. 379-396.

Lee, C. K., Tan, B. y Chlu, J. Z. (2008). The impact of organizational culture and learning on innovation performance. International Journal of Innovation and Learning, 5 (4), pp. 413-428.

Maldonado, G., Madrid, A., Martinez, M. y Aguilera, L. (2009). Los efectos de la innovación en el rendimiento de las MIPYMES de Aguascalientes: una evidencia empírica. Recuperado el 25 de junio del 2010, de: http:// www.revista.economia.uady.mx/2009/ $\mathrm{XXVI/73/02.pdf.}$

Manimala, M. J. (2006). Organizational Constraints on Innovation and Intrapreneurship: Insights from Public Sector. VIKALPA, n. 31, pp. 49-6o.

Martins, E. y Terblanche, F. (2003). Building organizational culture that stimulates creativity and Innovation. European Journal of Innovation Management, 6 (1), pp. 64-74.

Médina, C. y Espinoza, M. (1994). La innovación en las organizaciones modernas. Recuperado el 12 de junio del 2010, de: http://www.azc.uam.mx/ publicaciones/gestion/num5/doco6.htm. 
Moriano, J., Topa, G., Valero, E. y Levy, J. (2009). Identificación organizacional y conducta "intraemprendedora". Anales de Psicología, 25(2), pp. 277-287.

Morris, M. A, Avila, R. y Allen, J. (1993). Individualism and the modern coporation: Implicacions for innovation and entrepreneurship. Journal of management, 19(3), pp. 595-612.

Morris, M. y Kuratko, D. F. (2002). Corporate Entrepreneurship. Orlando, FL: Harcourt College Publishers.

Naranjo, J. C., Sanz, R. y Jiménez, D. (2008). Cultura organizacional e innovación: un estudio empírico. En J. P. García, Estableciendo puentes en una economía global. Salamanca, España: Asociación Europea de Dirección y Economía de Empresa.

OECD y EUROSTAT. (2005). Manual de Oslo. Oslo, Noruega: OECD.

Pinchot III, G. (1985). Intrapreneuring. New York, EUA: Harper y Row.

Ross, J. (1987). Intrapreneurship and Corporate Culture. Industrial Management , 29 (1), pp. 22-25.

Samuelsson, M. y Dahlqvist, J. (2005). Which Schumpeter is right? Comparing nascent exploitation processes. En P. D. Reynolds et al. (Eds.), Frontiers of entrepreneurial research pp. 1-10. Babson Park, FL: Babson College Center.

Sathe, V. (2003). Corporate entrepreneurship: top managers and new business creation. Cambridge, Inglaterra: Cambridge University Press.
Trujillo, M. A. y Guzmán, A. (2008). Intraemprendimiento: Una revisión al constructo teórico, sus implicaciones y agenda de investigación futura. Cuadernos de Administración, 21 (35), pp. 37-63.

Van-Auken, H., Madrid, A. y García, D. (2008). Innovation and performance in Spanish manufacturing SMEs. International Journal of Entrepreneurship and Innovation Management, 8 (1), pp. 36-56.

Van-Brusel, G. y Ulijn, J. (2008). Developing Intrapreneurship as a Career Perspective for Senior Professionals: Towards an Innovative HRM and Career Management Approach, en The 5th international conference on innovation and management. 10-11 de diciembre 2008. Maastricht, The Netherlands.

Varela, J. y Irizar, I. (2009). Caracterización de los Intraemprendimientos en el Grupo Mondragón de España y en las empresas de Ibagué en Colombia, Recuperado el 21 de enero de 2011 de: http://www.clee2008.ufsc.br/31.pdf.

World Economic Forum. (2009). Evaluación de la competitividad en el sector de viajes y turismo en Colombia. Washington D.C.: World Economic Forum.

Wood, C. (2004). Entrepreneurial Mindset in Department of Defense (DoD) Organizations Antecedents and Outcomes, Tesis de Maestría para la Air Force Institute of Technology. Recuperado el 19 de enero del 2011, de: http://www.dtic.mil/cgi-bin/ GetTRD oc? Location $=\mathrm{U}_{2} \& \mathrm{doc}=\mathrm{G}$ etTRD oc . pdf\&AD=ADA 423134

Zhao, F. (2005). Exploring the synergy between entrepreneurship and innovation. International Journal of Entrepreneurial Behaviour \& Research, 1 (1), pp. 25-41. 